\title{
In silico prediction of the T-cell and IgE-binding epitopes of Per a 6 and Bla g 6 allergens in cockroaches
}

\author{
HAO CHEN ${ }^{1,2^{*}}$, HAI-WEI YANG ${ }^{2 *}$, JI-FU WEI $^{1,2}$ and AI-LIN TAO ${ }^{1}$ \\ ${ }^{1}$ Guangdong Provincial Key Laboratory of Allergy and Clinical Immunology, The State Key Laboratory of \\ Respiratory Disease, The Second Affiliated Hospital of Guangzhou Medical University, Guangzhou, \\ Guangdong 510260; ${ }^{2}$ Research Division of Clinical Pharmacology, The First Affiliated Hospital of \\ Nanjing Medical University, Nanjing, Jiangsu 210029, P.R. China
}

Received August 26, 2013; Accepted April 2, 2014

DOI: $10.3892 / \mathrm{mmr} .2014 .2399$

\begin{abstract}
Per a 6 and Bla g 6 are cockroach allergens found in Periplaneta americana and Blattella germanica, respectively. The objective of the present study was to predict the B- and T-cell epitopes of the Per a 6 and Bla g 6 allergens. Three immunoinformatics tools, the DNAStar Protean system, the Bioinformatics Predicted Antigenic Peptides system and the BepiPred 1.0 server, were used to predict the potential B-cell epitopes, while Net-MHCIIpan-2.0 and NetMHCII-2.2 were used to predict the T-cell epitopes of the two allergens. As a result, seven peptides were predicted in the Per a 6 allergen and seven peptides were predicted in the Bla g 6 allergen in the B-cell epitope predictions. In the T-cell prediction, the Per a 6 allergen was predicted to have nine strongly binding nonamer core epitope sequences $\left(\mathrm{IC}_{50}<50 \mathrm{~nm}\right)$ and 28 weakly binding sequences $\left(50 \mathrm{~nm}<\mathrm{IC}_{50}<500 \mathrm{~nm}\right)$, while the Bla g 6 allergen was predicted to have nine strong binders and 25 weak binders. These results may be useful for peptide-based vaccine designs.
\end{abstract}

\section{Introduction}

Cockroach allergies are type I hypersensitivities mediated by immunoglobulin $\mathrm{E}$ ( $\mathrm{IgE}$ ) and have been recognized as

Correspondence to: Professor Ai-Lin Tao, Guangdong Provincial Key Laboratory of Allergy and Clinical Immunology, The State Key Laboratory of Respiratory Disease, The Second Affiliated Hospital of Guangzhou Medical University, 250 Gangdong Road, Guangzhou, Guangdong 510260, P.R. China

E-mail: taoailin@gzhmu.edu.cn

Professor Ji-Fu Wei, Research Division of Clinical Pharmacology, The First Affiliated Hospital, Nanjing Medical University, 300 Guangzhou Road, Nanjing, Jiangsu 210029, P.R. China

E-mail: weijifu@hotmail.com

*Contributed equally

Key words: Per a 6, Bla g 6, B-cell epitopes, T-cell epitopes, immunoinformatics prediction such since 1964 (1). Cockroach allergies are associated with the development of asthma and have been shown to put patients with asthma at risk of requiring emergency medical aid. This is a particular issue for asthmatic children in less affluent urban households, where cockroach infestations are more prevalent (2). Several proteins from cockroaches are potent environmental aeroallergens. Skin prick tests in China revealed that $25.7 \%$ of patients with allergies reacted positively to the American cockroach Periplaneta americana (Per a allergens) and $18.7 \%$ exhibited positive responses to the German cockroach Blattella germanica (Bla g allergens) (3). Among the indoor cockroaches, the American cockroach is the dominant species and is responsible for a large proportion of the cockroach-induced allergic reactions worldwide. Seven allergens from Periplaneta americana have been characterized: Per a 1 (4), Per a 2 (5), Per a 4 (6), Per a 6 (7), Per a 7 (8), Per a 9 (9) and Per a 10 (10). Per a 6 is a troponin C protein identified in the American cockroach. It was found that native Per a 6-bound IgE was present in the sera of $17 \%$ of Thai individuals with cockroach allergies, indicating that Per a 6 is a minor cockroach allergen (11).

To date, the use of cockroach immunotherapy has been rare and there are few reports on its efficacy $(12,13)$. Elucidation of the B- and T-cell epitopes of allergens may enhance the understanding of the structure-function relationship and predict the basis of cross-reactivity. Cross-reactive epitopes may be useful in reducing the number of allergens without compromising the efficacy of therapy (14). B-cell epitopes can be applied in the diagnosis, therapy and development of effective vaccines for immunotherapy. Numerous techniques can be utilized in the identification of B-cell epitopes; in particular, computational tools have been suggested to represent a promising and rapid approach. Following identification, the allergenicity of an allergen can be reduced by modifying the predicted B-cell epitopes (15). In the last decade, T-cell epitopes were successfully identified based on computer simulation. To stimulate T-lymphocyte responses, extracellular peptides bind to major histocompatibility complex (MHC) class II molecules (16). Therefore, T-cell epitopes were indirectly predicted by the identification of MHC-binding molecules (17). In the present study, the B- and T-cell epitopes of the Per a 6 allergen and its analog in the German cockroach, the Bla g 6.01 allergen, were identified by an in silico approach. The results indicated the 
potential utility of the B- and T-cell epitopes in a peptide-based vaccine design for cockroach allergy.

\section{Materials and methods}

Sequence retrieval. The primary sequence of the Per a 6 allergen was acquired from the nucleotide database of the National Center for Biotechnology Information (NCBI; http://www.ncbi.nlm.nih.gov/) with the accession number AY792950.1. The Basic Local Alignment Search Tool (BLAST; http://blast.ncbi.nlm.nih.gov/Blast.cgi) was used to obtain the homologous sequences.

Physiochemical analysis. Physiochemical analysis of the Per a 6 allergen and its homologous sequences, including analysis of the molecular weight, theoretical isoelectric point (pI), amino acid composition, instability index, aliphatic index and grand average of hydropathicity (GRAVY), was performed using the ProtParam tool (http://web.expasy.org/protparam/).

Phylogenetic analysis. The complete amino acid sequences of the Per a 6 allergen and its homologs were aligned using Clustalx 2.1 (18). The phylogenetic tree was obtained using the neighbor-joining ( $\mathrm{NJ}$ ) method on the basis of the Jones-Taylor-Thornton amino acid sequence distance implemented in the Molecular Evolutionary Genetics Analysis 5 software (19). The reliability was evaluated by the bootstrap method with 1,000 replications.

In silico prediction of $B$-cell epitopes. In the Protean ${ }^{\mathrm{TM}}$ system (DNAStar, Inc., Madison, WI, USA), four properties (hydrophilicity, flexibility, accessibility and antigenicity) of the amino acid sequences were selected as parameters for the prediction of epitopes. The hydrophilicity prediction was performed by the methods of Hopp and Woods (20) and Kyte and Doolittle (21). Flexibility was analyzed by the strategies of Karplus and Schulz (22), while the surface accessibility and antigenicity were analyzed by the strategies of Emini et al (23) and Jameson and Wolf (24). The peptide regions with good hydrophilicity, high flexibility, surface accessibility and a high antigenic index were selected as candidate epitopes for further investigation. In order to enhance the veracity of prediction towards the B-cell epitopes, the amino acid sequences were analyzed by the Protean system.

The Bioinformatics Predicted Antigenic Peptides (BPAP) system (http://imed.med.ucm.es/Tools/antigenic.pl) and BepiPred1.0server(http://www.cbs.dtu.dk/services/BepiPred/) were additionally used to predict B-cell epitopes. The BPAP system follows the prediction method by Kolaskar and Tongaonkar (25), while the BepiPred 1.0 server follows a combination of a hidden Markov model and a propensity scale method (26). The two servers only require the amino acid sequence and provide more straightforward results, which are combined with the physicochemical properties of the amino acids, including hydrophilicity, flexibility, accessibility, turns and an exposed surface (27). The ultimate consensus epitope results were obtained by combining the results of the three immunoinformatics tools with the previously published method (28). If all three methods concluded that the sequence was not an epitope, the consensus result was $0 \%$ epitope.
However, if only one or none of the methods predicted that the sequence was not an epitope, the consensus result was $67 \%$ or $100 \%$ epitope, respectively. The regions whose consensus epitope result was 67 or $100 \%$ were selected as the final potential epitope regions.

In silico prediction of T-cell epitopes. T-cell epitopes are principally predicted indirectly by identifying the binding of peptide fragments to the MHC complexes. However, the binding grooves of $\mathrm{MCH}-\mathrm{II}$ molecules are open at both ends, allowing various lengths of peptides to bind, and the same MHC molecule can accommodate various different binding sequences. These two properties make the development of accurate predictive algorithms for MHC-class II binding complicated. It has been reported that a sequence nine amino acids in length (9-mer), known as a core peptide, is essential for MHC-class II binding (29). For human leukocyte antigen (HLA)-DR-based T-cell epitope prediction, the artificial neural network-based alignment (NN-align) method NetMHCIIpan-2.0 (http://www.cbs.dtu. $\mathrm{dk} /$ services/NetMHCIIpan/) was applied. NetMHCIIpan-2.0 provides predictions only for a selection of the most commonly found HLA-DR alleles. For HLA-DQ alleles, NetMHCII-2.2 (http://www.cbs.dtu.dk/services/NetMHCII/) was used (30). Although the predicted results provided limited binding-affinity data, it was recently reported to provide the best performance in predicting this locus (31). For the T-cell prediction process, the peptides predicted to be significant binders by at least one allele were examined. The binding significance of each peptide to the given MHC molecule was based on the estimated strength of binding exhibited by a predicted nested core peptide at a set threshold level. Based on the estimated strength of binding, the peptides were characterized as significant (weak or strong) or non-significant. Each predicted core 9-mer sequence represented the predicted putative T-cell epitope within each peptide examined (32).

\section{Results}

Physiochemical analysis and phylogenetic analysis. The amino acid sequence of the Per a 6 allergen was obtained from the nucleotide database of the NCBI. The primary structure contained 151 amino acids. A search with BLAST obtained 16 homologous sequences of the allergen. The accession numbers of these homologs were DQ279092.1 (Blattella germanica allergen Bla g 6.01), DQ279093.1 (Blattella germanica allergen Bla g 6.02), XM_001987063 (Drosophila grimshawi GH21731), XM_002049988 (Drosophila virilis troponinC41C), XM_002106068.1 (Drosophila simulans GD16679), XM_002086000 (Drosophila yakuba GE11315), GQ396705.1 (Bombyx mori troponin CIII), XM_001688761.1 (Anopheles gambiae str. PEST), AY075534.1 (Drosophila melanogaster RH0783), NM_078895.4 (Drosophila melanogaster troponin C), X76043.1 (Drosophila melanogaster troponin C), XM_002063468.1 (Drosophila willistoni GK21371), AB180453.1 (Plutella xylostella troponin C), XM_001867954.1 (Culex quinquefasciatus troponin C), AF432912.1 (Solenopsis invicta troponin C), and NM_001205104 
Table I. Physiochemical properties of the Per a 6 allergen and its homologous sequences.

\begin{tabular}{|c|c|c|c|c|c|c|c|}
\hline Accession no. & MW & NCR (n) & PCR (n) & $\mathrm{TP}$ & II & AI & G \\
\hline AY792950.1 & 17130.9 & 44 & 11 & 3.84 & 24.97 & 87.22 & -0.377 \\
\hline DQ279092.1 & 17216.2 & 43 & 13 & 3.96 & 35.44 & 86.56 & -0.388 \\
\hline DQ279093.1 & 17095.1 & 43 & 13 & 3.96 & 31.48 & 87.22 & -0.269 \\
\hline XM_001987063 & 16989.7 & 40 & 10 & 3.84 & 32.11 & 86.05 & -0.324 \\
\hline XM_002049988 & 17557.4 & 40 & 10 & 3.84 & 29.52 & 81.58 & -0.317 \\
\hline XM_002106068.1 & 17155.9 & 40 & 10 & 3.83 & 31.63 & 83.05 & -0.325 \\
\hline XM_002086000 & 16937.6 & 40 & 10 & 3.83 & 29.02 & 84.14 & -0.336 \\
\hline GQ396705.1 & 17275.3 & 42 & 13 & 4.03 & 34.45 & 86.01 & -0.291 \\
\hline XM_001688761.1 & 17609.5 & 42 & 12 & 3.90 & 27.87 & 85.00 & -0.384 \\
\hline AY075534.1 & 17155.9 & 40 & 10 & 3.83 & 31.63 & 83.05 & -0.325 \\
\hline NM_078895.4 & 17155.9 & 40 & 10 & 3.83 & 31.63 & 83.05 & -0.325 \\
\hline X76043.1 & 17125.9 & 39 & 11 & 3.90 & 31.77 & 82.94 & -0.345 \\
\hline XM_002063468.1 & 17036.9 & 37 & 11 & 3.99 & 34.09 & 87.22 & -0.220 \\
\hline AB180453.1 & 17262.2 & 42 & 13 & 4.05 & 37.38 & 84.12 & -0.322 \\
\hline XM_001867954.1 & 18057.9 & 40 & 11 & 3.99 & 28.80 & 83.60 & -0.343 \\
\hline AF432912.1 & 17304.2 & 42 & 13 & 4.01 & 38.57 & 84.84 & -0.457 \\
\hline NM_001205104 & 17720.5 & 45 & 12 & 3.84 & 34.45 & 85.82 & -0.394 \\
\hline
\end{tabular}

MW, molecular weight; NCR, negatively charged residues; PCR, positively charged residues; TP, theoretical pI; II, instability index; AI, aliphatic index; G, gravity; AY792950.1, Per a 6 allergen; DQ279092.1, Bla g 6.01 allergen; DQ279093.1, Bla g 6.02 allergen.

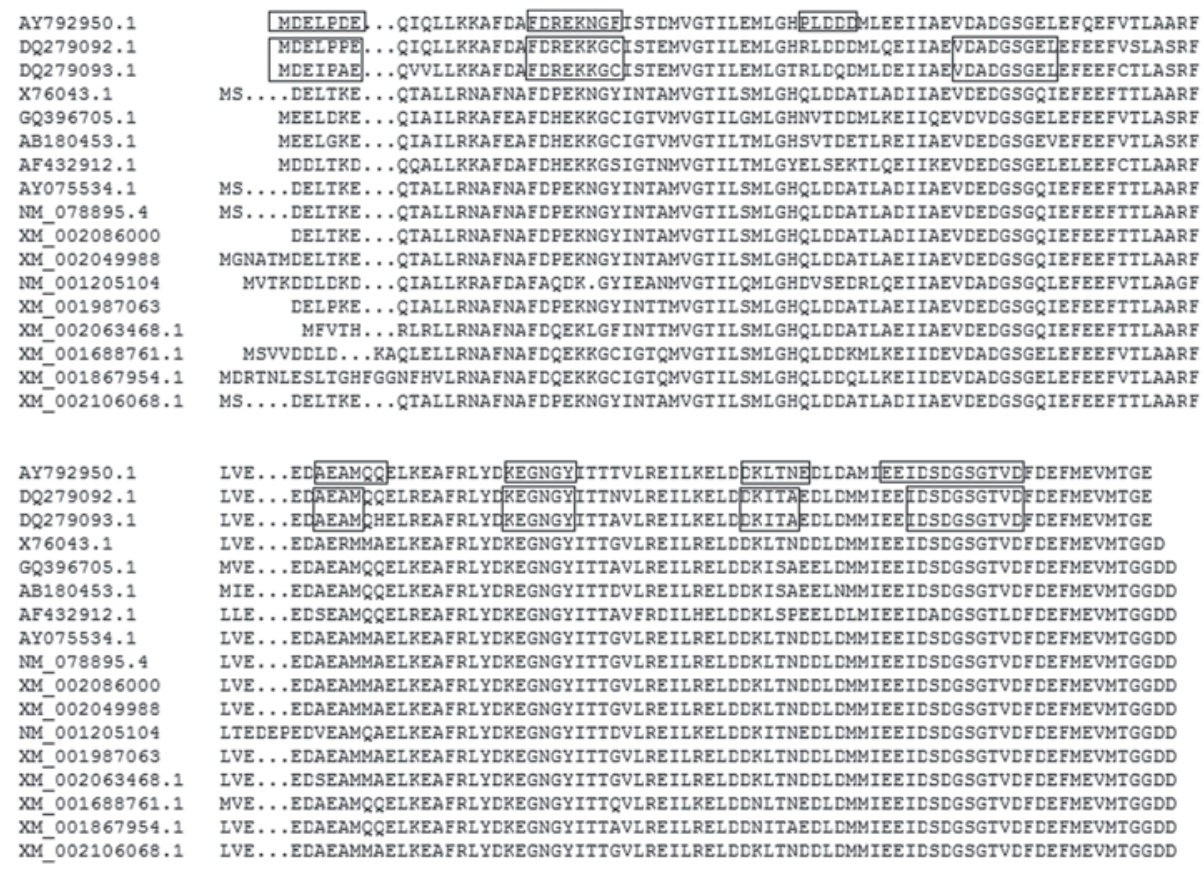

Figure 1. Alignment of the Per a 6 allergen and its homologs. The predicted peptides are displayed in gray boxes. AY792950.1, Per a 6 allergen; DQ279092.1, Bla g 6.01 allergen; DQ279093.1, Bla g 6.02 allergen.

(Acyrthosiphon pisum troponin C-like). Table I shows the details of the physiochemical analysis of these homologs. All homologs were stable (instability index ranging between 24.97 and 38.57) with theoretical pIs ranging between 3.83 and 4.05. The GRAVY values were found to be negative $(-0.457$ to -0.220$)$ for complete sequences.
The alignment of the Per a 6 allergen and its homologs is shown in Fig. 1. The evolutionary tree (Fig. 2) was inferred by the NJ method. Phylogenetic analysis showed that Bla g 6.01 (DQ279092.1) and Bla g 6.02 (DQ279093.1) were two related sequences that were similar to the Per a 6 allergen. Bla g 6.01 and Bla g 6.02 are two cockroach isoallergens identified from 
Table II. Location of the B-cell epitopes of the Per a 6 allergen predicted by three immunoinformatics tools.

\begin{tabular}{ll}
\hline Tools & Location of the prediction results \\
\hline DNAStar Protean & $1-7,17-27,42-48,81-89,96-102$, \\
& $114-123,129-140$ \\
BPAP & $5-16,32-41,49-55,64-78,103-113$ \\
BepiPred 1.0 server & $1-7,19-26,42-46,81-86,97-103$, \\
& $117-122,131-140$
\end{tabular}

BPAP, Bioinformatics Predicted Antigenic Peptides.

Table III. Location of the B-cell epitopes of the Bla g 6.01 allergen predicted by three immunoinformatics tools.

\begin{tabular}{ll}
\hline Tools & Location of the prediction results \\
\hline DNAStar Protean & $1-7,17-27,42-47,55-65,81-91$, \\
& $95-102,113-122,129-140$ \\
BPAP & $5-15,23-29,32-41,48-54,65-78$, \\
& $107-113$ \\
BepiPred 1.0 server & $1-7,19-26,55-63,81-84,97-103$, \\
& $117-121,131-140$
\end{tabular}

BPAP, Bioinformatics Predicted Antigenic Peptides.

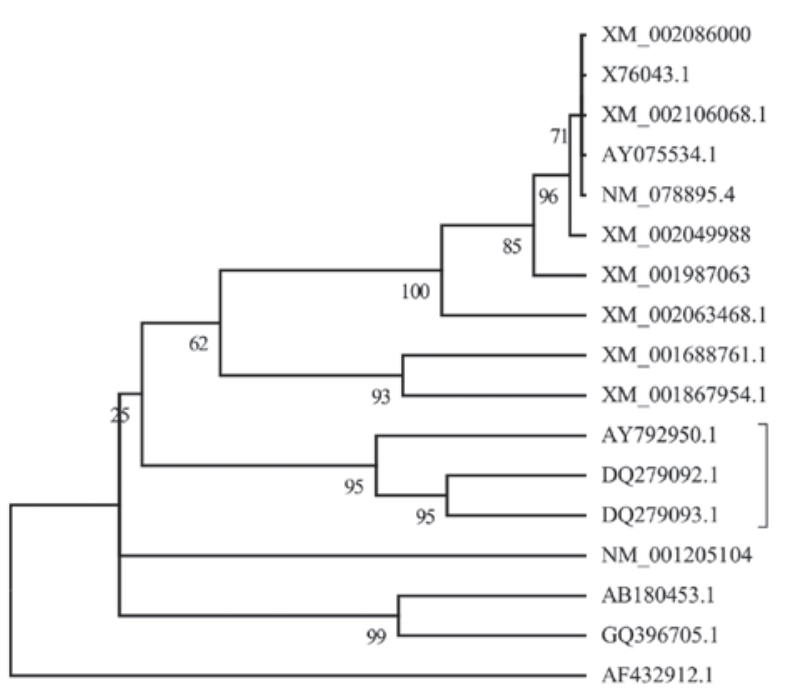

Figure 2. Phylogenetic association of the Per a 6 allergen amino acid sequence with other homologs. Bla g 6.01 and Bla g 6.02 are two related sequences similar to Per a 6. AY792950.1, Per a 6 allergen; DQ279092.1, Bla g 6.01 allergen; DQ279093.1, Bla g 6.02 allergen.

Blatella germanica, with Bla g 6.01 exhibiting 93\% amino acid identity to Bla g 6.02. In the present study, the B- and T-cell epitopes of the Per a 6 and Bla g 6.01 allergens were investigated.

\section{Epitope prediction}

$B$-cell epitope prediction. Surface accessibility and fragment flexibility are important features for predicting antigenic
Table IV. B-cell epitopes of the Per a 6 allergen predicted by combined immunoinformatics methods.

\begin{tabular}{lccl}
\hline Number & Position & $\begin{array}{c}\text { Length of } \\
\text { sequence } \\
\text { (amino acids) }\end{array}$ & \multicolumn{1}{c}{ Sequence } \\
\hline Peptide 1 & $1-7$ & 7 & MDELPDE \\
Peptide 2 & $19-26$ & 8 & FDREKNGF \\
Peptide 3 & $42-46$ & 5 & PLDDD \\
Peptide 4 & $81-86$ & 6 & AEAMQQ \\
Peptide 5 & $97-102$ & 6 & KEGNGY \\
Peptide 6 & $117-122$ & 6 & DKLTNE \\
Peptide 7 & $129-140$ & 12 & EEIDSDGSGTVD \\
\hline
\end{tabular}

Table V. B-cell epitopes of the Bla g 6 allergen predicted by combined immunoinformatics methods.

\begin{tabular}{|c|c|c|c|}
\hline Number & Position & $\begin{array}{c}\text { Length of } \\
\text { sequence } \\
\text { (amino acids) }\end{array}$ & Sequence \\
\hline Peptide 1 & $1-7$ & 7 & MDELPDE \\
\hline Peptide 2 & $19-26$ & 8 & FDREKKGC \\
\hline Peptide 3 & $55-63$ & 9 & VDADGSGEL \\
\hline Peptide 4 & $81-84$ & 4 & AEAM \\
\hline Peptide 5 & $97-102$ & 6 & KEGNGY \\
\hline Peptide 6 & $117-121$ & 5 & DKITA \\
\hline Peptide 7 & $131-140$ & 10 & IDSDGSGTVD \\
\hline
\end{tabular}

epitopes. In addition, the existence of regions with high hydrophobicity provides strong evidence for epitope identification. As shown in Figs. 3 and 4, the hydrophilic regions occupied most of the sequence, indicating high hydrophilicity of the Per a 6 and Bla g 6.01 allergens. Furthermore, the frequent appearance of the surface and flexibility regions indicated that the Per a 6 and Bla g 6.01 sequences were flexible, stretched and easily exposed to the outside. The stretched structure was further validated by the results of the flexibility and surface accessibility analysis. The antigenic index directly showed the epitope-forming capacity of the Per a 6 and Bla g 6.01 sequences. Most of the sequences exhibited a high antigenic index, suggesting a high tendency of Per a 6 and Bla g 6.01 to form epitopes. Based on these sequence properties, the final regions predicted by DNAStar Protean were obtained as shown in Tables II and III. Tables II and III also show the predicted results obtained using the BPAP system and the BepiPred 1.0 server. The final potential epitopes of the Per a 6 and Bla g 6.01 allergens were selected on the basis of the results from these two tools and those from the DNAStar Protean system. The ultimate results of the three immunoinformatics tools predicted seven peptides (1-7, 19-26, 42-46, 81-86, 97-102, 117-122 and 129-140) to be the potential linear B-cell epitopes of the Per a 6 allergen, and seven peptides, 1-7, 19-26, 55-63, 81-84, 97-102, 117-121 and 131-140, were predicted as 
Table VI. Location of the T-cell epitopes of the Per a 6 allergen predicted by Net-MHCIIpan-2.0 and NetMHCII-2.2.

Per a 6 allergen

Strong-binding core peptides

Weak-binding core peptides
$7-15,35-43,65-73,66-74,68-76,102-110,121-129,123-131,142-150$

9-17, 12-20, 26-34, 27-35, 28-36, 31-39, 32-40, 33-41, 36-44,47-55, 48-56, 51-59, 63-71, 64-72, 69-77, 75-83, 84-92, 86-94, 87-95, 88-96, 94-102, 95-103, 100-108, 103-111, 107-115, $111-119,120-128,140-148$

Strong-binding, $\mathrm{IC}_{50}<50 \mathrm{~nm}$; weak-binding, $50 \mathrm{~nm}<\mathrm{IC}_{50}<500 \mathrm{~nm}$.

Table VII. Location of the T-cell epitopes of the Bla g 6 allergen predicted by Net-MHCIIpan-2.0 and NetMHCII-2.2.

Bla g 6.01 allergen

Strong-binding core peptides

Weak-binding core peptides
$8-16,35-43,50-58,68-76,75-83,84-92,88-96,89-97,142-150$

4-12, 10-18, 12-20, 28-36, 24-32, 26-34, 41-49, 45-53, 48-56, 51-59, 65-73, 69-77, 70-78

73-81, 76-84, 77-85, 86-94, 95-103, 100-108, 102-110, 107-114, 108-116, 111-119, 119-127, $139-147$

Strong-binding, $\mathrm{IC}_{50}<50 \mathrm{~nm}$; weak-binding, $50 \mathrm{~nm}<\mathrm{IC}_{50}<500 \mathrm{~nm}$.

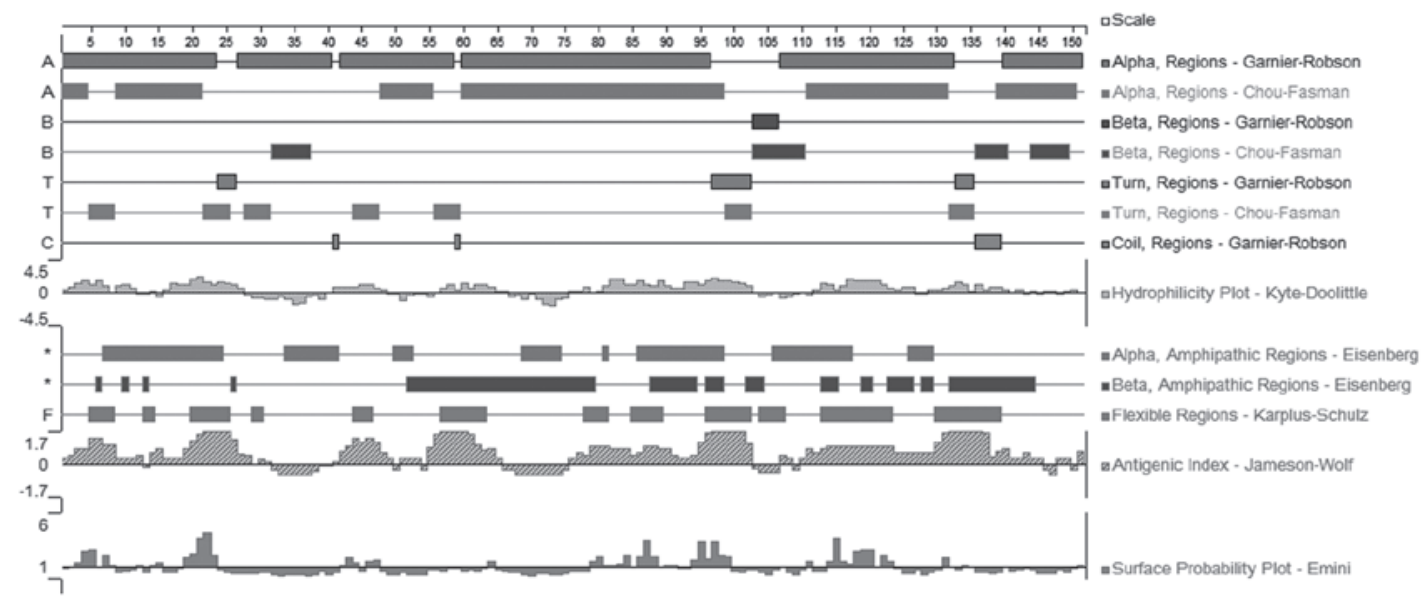

Figure 3. Analysis results of the amino acid sequence of the Per a 6 allergen using the DNAStar Protean system.

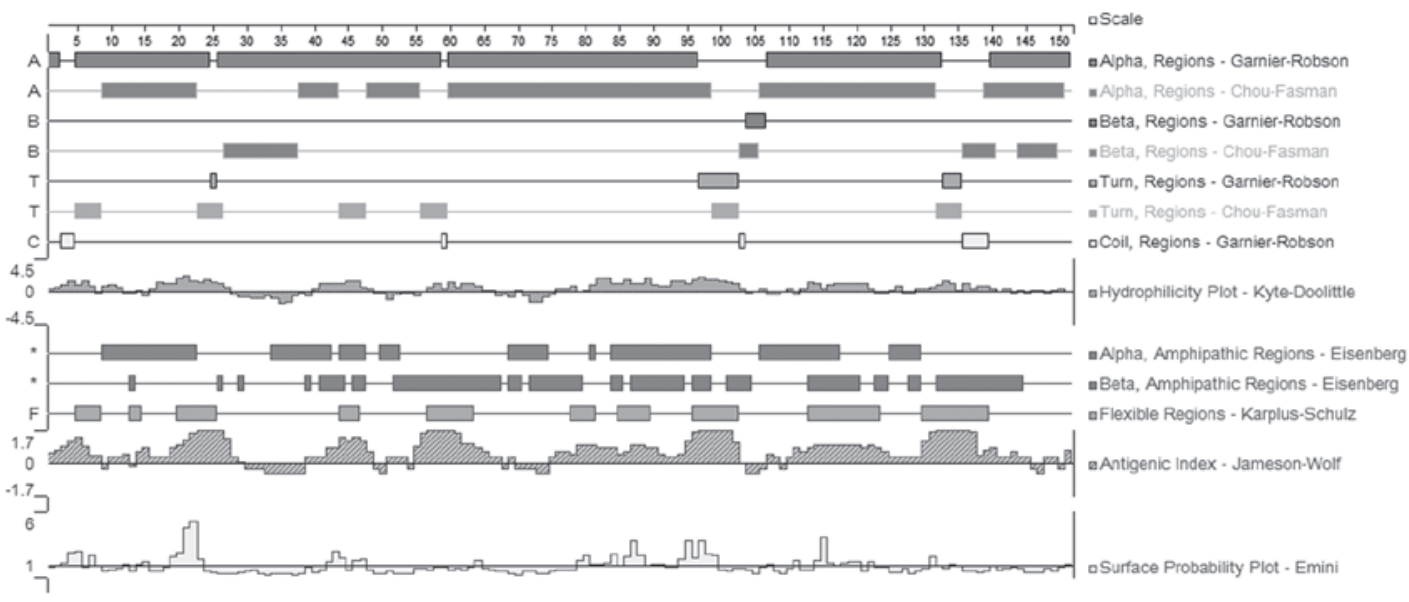

Figure 4. Analysis results of the amino acid sequence of the Bla g 6 allergen using the DNAStar Protean system. 
potential linear B-cell epitopes of the Bla g 6.01 allergen. The amino acid sequences are shown in Tables IV and V.

T-cell epitope prediction. Net-MHCIIpan-2.0 and NetMHCII-2.2 were used to identify significant core 9-mer peptides in the Per a 6 and Bla g 6.01 allergens. All estimations were based on the HLA-DRB1 alleles found in the study cohort. For HLA-DQ alleles, only DQB1 0201, 0402, 0501 and 0602 were used for epitope prediction. In the overall analysis using Net-MHCIIpan-2.0 and NetMHCII-2.2, Per a 6 was predicted to have nine strongly binding 9-mer core epitope sequences $\left(\mathrm{IC}_{50}<50 \mathrm{nM}\right)$ : Peptides 7-15, 35-43, 65-73, 66-74, 68-76, 102-110, 121-129, 123-131, 142-150 (Table VI). The Per a 6 allergen was also predicted to have 28 weakly binding 9-mer core epitope sequences $\left(50 \mathrm{~nm}<\mathrm{IC}_{50}<500 \mathrm{~nm}\right.$ ) (Table VI). The Bla g 6.01 allergen was predicted to have 34 significant nonamer core epitope sequences. Nine of them were strong binders and comprised peptides 8-16, 35-43, 50-58, 68-76, 75-83, 84-92, 88-96, 89-97 and 142-150 (Table VII). The remaining peptides were weak binders and are shown in Table VII.

\section{Discussion}

The prevalence and morbidity of allergies, such as asthma, rhinitis and eczema, in children have been increasing over recent decades. This is currently a major concern for the international medical community and cannot solely be attributed to the improved diagnosis of these diseases (33). Conventional immunotherapy for allergies using crude allergen extracts has been shown to have a high risk of anaphylaxis. One alternative approach is the use of peptide fragments corresponding to the B- and T-cell epitopes of the allergen (34). In a previous study, overlapping synthetic peptides were used to validate the IgE-binding capacity. Although this method decreases the possibility of missed epitopes, it requires the synthesis of large numbers of peptides, which is time-consuming and costly (35). In silico prediction has become a common and useful tool for selecting epitopes from immunologically relevant proteins, and can save the time and cost of peptide synthesis (36).

Per a 6 is a minor cockroach allergen from Periplaneta americana, while Bla g 6 is an allergen from Blatella germanica with a sensitization prevalence of $14 \%$ among patients with cockroach allergy. Bla g 6 consists of three isoallergens: Bla g 6.01, 6.02 and 6.03. Bla g 6.01 shows $93 \%$ amino acid identity to Bla g 6.02 and $68 \%$ identity to Bla g 6.03. The evolutionary tree in the present study (Fig. 2) showed that Bla g 6.01 and 6.02 are homologs of Per a 6 (11). To the best of our knowledge, the present study is the first to predict B- and T-cell epitopes from the Per a 6 and Bla g 6.01 allergens. A previous study showed that the prediction of B-cell epitopes using the bioinformatics approach correlated well with the experimental approach (37). The prediction of B-cell epitopes among protein sequences has been enabled by the development of numerous algorithms that are based on certain amino acid properties, including hydrophilicity, antigenicity, segmental mobility, flexibility and accessibility (38). In the present study, the linear B-cell epitopes of the Per a 6 and Bla g 6.01 allergens were predicted using three sequence-based tools (the DNAStar Protean system, BPAP and the BepiPred
1.0 server). Seven B-cell epitopes of Per a 6 and seven epitopes of Bla g 6 were investigated. The alignment results (Fig. 1) showed that all the peptides of Per a 6 and Bla $g$ were located in similar positions, with exception of peptide three. This indicated that Per a 6 shares a cross-reactive epitope with Bla g 6 . Peptide three of Per a 6 was located at position 42-46, while that of Bla g 6 was located at 55-63, indicating that peptide three may represent a distinction between the epitopes of these two cockroach allergens.

In recent years, certain algorithms have substantially improved in their accuracy to predict T-cell epitopes. However, most algorithms have targeted HLA-DR molecules, not HLA-DP or HLA-DQ molecules, despite the importance of HLA-DP and HLA-DQ molecules for antigen presentation. NetMHCpan-2.0 was recently shown to have a per-allele mean accuracy of 0.854 (1.0 being $100 \%$ accurate and 0.5 of no significance) (39). In another study, the per-allele mean accuracy of the NN-align method was 0.882 (31). In the present study, Net-MHCIIpan-2.0 and NetMHCII-2.2 were used to predict the core 9-mer T-cell epitopes in the Per a 6 and Bla g 6 allergens. Combining the results obtained with Net-MHCIIpan-2.0 and NetMHCII-2.2, a total of 37 and 34 epitopes were predicted for the Per a 6 and Bla g 6 allergens, respectively. Nine epitopes from the Per a 6 allergen and nine from Bla g 6 allergen were strongly binding, with an $\mathrm{IC}_{50}<50 \mathrm{~nm}$.

In conclusion, the present study predicted B- and T-cell epitopes of the Per a 6 allergen and its homolog, the Bla g 6.01 allergen in Blatella germanica, using an in silico method. The results may be used to benefit allergen immunotherapies and reduce the frequency of allergic reactions. However, the accuracy of these epitopes requires confirmation in further experiments.

\section{Acknowledgements}

The present study was sponsored by the National Natural Science Foundation of China (nos. 31340073, 81273274, 81373128, 810001329 and 30972822), the Great Project (no. 2011ZX08011-005) from the Major Program of National Science and Technology of China and the Special Research Project (no. 201300000159) from the Science and Information Technology of Guangzhou. National Major Scientific and Technological Special Project for 'Significant New Drugs Development' (2011ZX09302-003-02), and a project funded by the Priority Academic Development Program of Jiangsu Higher Education Institutions.

\section{References}

1. Bernton HS and Brown H: Insect allergy - preliminary studies of the cockroach. J Allergy 35: 506-513, 1964.

2. Arruda LK, Vailes LD, Ferriani VP, Santos AB, Pomés A and Chapman MD: Cockroach allergens and asthma. J Allergy Clin Immunol 107: 419-428, 2001.

3. Sun BQ, Lai XX, Gjesing B, Spangfort MD and Zhong NS: Prevalence of sensitivity to cockroach allergens and $\operatorname{IgE}$ cross-reactivity between cockroach and house dust mite allergens in Chinese patients with allergic rhinitis and asthma. Chin Med J (Engl) 123: 3540-3544, 2010.

4. He S, Zhang Z, Zhang H, et al: Analysis of properties and proinflammatory functions of cockroach allergens Per a 1.01s. Scand J Immunol 74: 288-295, 2011. 
5. Pan QR, Wang SM, Shang HS and Chew FT: Identification and characterization of Per a 2, the Bla g 2 allergen homologue from American cockroach (Periplaneta americana). J Allergy Clin Immunol 117: S115, 2006

6. Tan YW, Chan SL, Ong TC, et al: Structures of two major allergens, Bla g 4 and Per a 4, from cockroaches and their IgE binding epitopes. J Biol Chem 284: 3148-3157, 2009.

7. Khantisitthiporn $\mathrm{O}$, Sookrung $\mathrm{N}$, Tungtrongchitr A, et al: Native troponin-T of the American cockroach (CR), Periplaneta americana, binds to $\mathrm{IgE}$ in sera of $\mathrm{CR}$ allergic Thais. Asian Pac J Allergy Immunol 25: 189-197, 2007.

8. Asturias JA, Gómez-Bayón N, Arilla MC, et al: Molecular characterization of American cockroach tropomyosin (Periplaneta americana allergen 7), a cross-reactive allergen. J Immunol 162: 4342-4348, 1999.

9. Sookrung N, Chaicumpa W, Tungtrongchitr A, et al: Periplaneta americana arginine kinase as a major cockroach allergen among Thai patients with major cockroach allergies. Environ Health Perspect 114: 875-880, 2006.

10. Sudha VT, Arora N, Gaur SN, Pasha S and Singh BP: Identification of a serine protease as a major allergen (Per a 10) of Periplaneta americana. Allergy 63: 768-776, 2008.

11. Sookrung $\mathrm{N}$ and Chaicumpa W: A revisit to cockroach allergens. Asian Pac J Allergy Immunol 28: 95-106, 2010.

12. Kang BC, Johnson J, Morgan C and Chang JL: The role of immunotherapy in cockroach asthma. J Asthma 25: 205-218, 1988.

13. Srivastava D, Gaur SN, Arora $N$ and Singh BP Clinico-immunological changes post-immunotherapy with Periplaneta americana. Eur J Clin Invest 41: 879-888, 2011.

14. Sharma V, Singh BP, Gaur SN, Pasha S and Arora N: Bioinformatics and immunologic investigation on $\mathrm{B}$ and $\mathrm{T}$ cel epitopes of Cur I 3, a major allergen of Curvularia lunata. J Proteome Res 8: 2650-2655, 2009.

15. Wang HW, Lin YC, Pai TW and Chang HT: Prediction of B-cell linear epitopes with a combination of support vector machine classification and amino acid propensity identification. J Biomed Biotechnol 2011: 432830, 2011.

16. Jiminez-Lopez JC, Kotchoni SO, Rodríguez-García MI and Alché JD: Structure and functional features of olive pollen pectin methylesterase using homology modeling and molecular docking methods. J Mol Model 18: 4965-4984, 2012.

17. Nielsen M, Lund O, Buus S and Lundegaard C: MHC class II epitope predictive algorithms. Immunology 130: 319-328, 2010.

18. Larkin MA, Blackshields G, Brown NP, et al: Clustal W and Clustal X version 2.0. Bioinformatics 23: 2947-2948, 2007.

19. Kumar S, Nei M, Dudley J and Tamura K: MEGA: a biologist-centric software for evolutionary analysis of DNA and protein sequences. Brief Bioinform 9: 299-306, 2008.

20. Hopp TP and Woods KR: Prediction of protein antigenic determinants from amino acid sequences. Proc Natl Acad Sci USA 78: 3824-3828, 1981

21. Kyte J and Doolittle RF: A simple method for displaying the hydropathic character of a protein. J Mol Biol 157: 105-132, 1982.
22. Karplus PA and Schulz GE: Prediction of chain flexibility in proteins. Naturwissenschaften 72: 212-213, 1985.

23. Emini EA, Hughes JV, Perlow D and Boger J: Induction of hepatitis A virus-neutralizing antibody by a virus-specific synthetic peptide. J Virol 55: 836-839, 1985.

24. Jameson B and Wolf $H$ : The antigenic index: a novel algorithm for predicting antigenic determinants. Comput Appl Biosci 4: 181-186, 1988.

25. Kolaskar A and Tongaonkar PC: A semi-empirical method for prediction of antigenic determinants on protein antigens. FEBS Lett 276: 172-174, 1990.

26. Larsen JE, Lund $\mathrm{O}$ and Nielsen M: Improved method for predicting linear B-cell epitopes. Immunome Res 2: 2, 2006.

27. Zheng LN, Lin H, Pawar R, Li ZX and Li MH: Mapping IgE binding epitopes of major shrimp (Penaeus monodon) allergen with immunoinformatics tools. Food Chem Toxicol 49: 2954-2960, 2011

28. Yang $\mathrm{X}$ and $\mathrm{Yu} \mathrm{X}$ : An introduction to epitope prediction methods and software. Rev Med Virol 19: 77-96, 2009.

29. Rammensee HG, Friede T and Stevanoviíc S: MHC ligands and peptide motifs: first listing. Immunogenetics 41: 178-228, 1995.

30. Nielsen $\mathrm{M}$ and Lund O: NN-align. An artificial neural network-based alignment algorithm for MHC class II peptide binding prediction. BMC Bioinformatics 10: 296, 2009.

31. Wang P, Sidney J, Kim Y, et al: Peptide binding predictions for HLA DR, DP and DQ molecules. BMC Bioinformatics 11: 568, 2010.

32. Pascal M, Konstantinou GN, Masilamani M, Lieberman J and Sampson HA: In silico prediction of Ara h $2 \mathrm{~T}$ cell epitopes in peanut-allergic children. Clin Exp Allergy 43: 116-127, 2013.

33. Baena-Cagnani CE, Serra H, Teijeiro A and Croce JS: Prevention of allergy and asthma. Clin Exp Allergy Rev 3: 51-57, 2003.

34. Moldaver D and Larché M: Immunotherapy with peptides. Allergy 66: 784-791, 2011.

35. Lin J, Bardina L, Shreffler WG, et al: Development of a novel peptide microarray for large-scale epitope mapping of food allergens. J Allergy Clin Immunol 124: 315-322, 2009.

36. Li GF, Wang Y, Zhang ZS, et al: Identification of immunodominant Th1-type T cell epitopes from Schistosoma japonicum $28 \mathrm{kDa}$ glutathione-S-transferase, a vaccine candidate. Acta Biochim Biophys Sin (Shanghai) 37: 751-758, 2005.

37. Nair S, Kukreja N, Singh BP and Arora N: Identification of B cell epitopes of alcohol dehydrogenase allergen of Curvularia lunata. PLoS One 6: e20020, 2011.

38. Pomés A: Relevant B cell epitopes in allergic disease. Int Arch Allergy Immunol 152: 1-11, 2010.

39. Nielsen M, Justesen S, Lund O, Lundegaard C and Buus S: NetMHCIIpan-2.0 - Improved pan-specific HLA-DR predictions using a novel concurrent alignment and weight optimization training procedure. Immunome Res 6: 9, 2010. 